\title{
INVESTIGATING INTEROPERABILITY CAPABILITIES BETWEEN IFC AND CITYGML LOD 4 - RETAINING SEMANTIC INFORMATION
}

\author{
G. S. Floros ${ }^{1,2} *$, C. Ellul ${ }^{2}$, E. Dimopoulou ${ }^{1}$ \\ ${ }^{1}$ School of Rural and Surveying Engineering, National Technical University of Athens, 9 Iroon Polytechneiou str., 15780 Zografou, \\ Athens, Greece - flwrosg@gmail.com; efi@survey.ntua.gr \\ ${ }^{2}$ Dept. of Civil, Environmental \& Geomatic Engineering, UCL, Gower Street, London, WC1E 6BT, UK - c.ellul@ucl.ac.uk
}

KEY WORDS: CityGML, IFC, 3D Model, BIM, GIS, Data Integration, FME

\begin{abstract}
:
Applications of 3D City Models range from assessing the potential output of solar panels across a city to determining the best location for $5 \mathrm{G}$ mobile phone masts. While in the past these models were not readily available, the rapid increase of available data from sources such as Open Data (e.g. OpenStreetMap), National Mapping and Cadastral Agencies and increasingly Building Information Models facilitates the implementation of increasingly detailed 3D Models. However, these sources also generate integration challenges relating to heterogeneity, storage and efficient management and visualization. CityGML and IFC (Industry Foundation Classes) are two standards that serve different application domains (GIS and BIM) and are commonly used to store and share 3D information. The ability to convert data from IFC to CityGML in a consistent manner could generate 3D City Models able to represent an entire city, but that also include detailed geometric and semantic information regarding its elements. However, CityGML and IFC present major differences in their schemas, rendering interoperability a challenging task, particularly when details of a building's internal structure are considered (Level of Detail 4 in CityGML). The aim of this paper is to investigate interoperability options between the aforementioned standards, by converting IFC models to CityGML LoD 4 Models. The CityGML Models are then semantically enriched and the proposed methodology is assessed in terms of model's geometric validity and capability to preserve semantics.
\end{abstract}

\section{INTRODUCTION}

3D City Models underpin tasks ranging from solar panel placement analysis to noise and air quality modelling and on to cadastral mapping (Open Geospatial Consortium, 2012). Beyond these standard functions, the rapid emergence of the Internet of Things, sensors and Smart Cities now also require increasingly detailed 3D City Models to maximize their effectiveness, as the data streams from these sources cannot be integrated and analyzed without modelling their location in 3D space (Batty et al., 2012). Two main sources of data for the required $3 \mathrm{D}$ models can be identified - Geographical Information Systems, and, more recently, Building Information Models.

A cursory glance may suggest that these two sources of data can appear similar- they both model the built environment, both fields are applied for similar application purposes, such as planning, asset management, infrastructure and buildings functionality (Ohori et al., 2017). However, their intended purpose is in fact very different. Geographical Information Systems (GIS) focuses on the spatial relationships between the different features of a city model, while Building Information Modelling (BIM) delves into the building process of the model and its structural characteristics in great detail. Moreover, the main characteristic of GIS is that the model is geolocated and often analysis is approached from a geographic perspective, while BIM facilitates construction projects, thus focusing on a building/architectural perspective, providing more internal detail.

Data interoperability between the two sources can facilitate a smoother communication between different types of professionals, as well as a more efficient absorption, storage and management of the raw data produced by sources such as mobile phones. Additionally, the similarity in application purposes between GIS and BIM highlight the potential for one domain to act as a data source for the other. To address those challenges, in this paper, two dominant data modelling standards are examined, one from each domain. CityGML, a 3D GIS open data model for the exchange of 3D City Models and Industry Foundation Classes (IFC) which describes the life-cycle of a building (Building SMART International, 2007). Significant challenges for interoperability options between CityGML and IFC derive from different kind of users and application fields that each Standard address, different handling of geometry and semantics, different representations and scaling, different focus on the objects of physical reality and different methods of data storage and management (Liu, 2017).

\subsection{Research Questions}

Review of existing work (Boyes et al., 2017; Ohori et al., 2017; Jusuf et al., 2017) demonstrates the potential of software such as Safesoft's FME to directly convert data from IFC to CityGML. However, manual intervention is required, and the work carried out focusses on building structure rather than interior detail (LoD3 in CityGML). This paper aims to extend the current state of the art by proposing a conversion workflow as a step forward in integrating 3D GIS and BIM by focusing on addressing: a) limitations regarding geometric issues with particular focus on LoD 4 models and b) facilitating a lossless semantic conversion and transfer throughout a semi-automatic process. For the latter, the preservation of semantics is examined both for information that is stored in geometric objects, such as the material of the wall and information that is stored in non-geometric objects such as the legal description of a building. Within that context, the capability of BIM to preserve this type of information when exporting to IFC and 
converting to CityGML is also examined. The proposed methodology is tested on two models utilizing digital architectural as-built drawings and the following research questions are addressed:

1. How can a BIM model be created in order to best enable downstream use in CityGML LoD 4 ?

2. To what extent can the conversion process be automated, while preserving and transferring both semantic and geometric information?

In particular, we attempt these tasks using off-the-shelf software to highlight some limitations of current tools and demonstrate that this type of conversion is not a solved problem if using off-the-shelf tools.

\subsection{Outline}

The paper is structured as follows: firstly, an analysis of the state of the art in 3D data integration is performed. Secondly an overview of the data and software is presented, followed by a detailed explanation of the developed methodology. Key findings of the research are summarized and the developed methodology is evaluated based on the generated models and the research questions that are originally set. Finally, conclusions and areas of future research are proposed.

\section{STATE OF THE ART IN BIM/3D GIS DATA INTEGRATION}

\subsection{Brief overview of CityGML and IFC}

CityGML is an open data model, based on XML format for storing, managing and exchanging virtual $3 \mathrm{D}$ city models (Open Geospatial Consortium, 2012, p. 9). CityGML standard supports 5 different Levels of Detail (LoD) which aim to facilitate an effective visualization and an efficient spatial analysis of the 3D models (Open Geospatial Consortium, 2012, p. 11). The Building model is currently the most detailed thematic extension of CityGML and consists of boundary surfaces, openings, rooms and building installations. CityGML can be extended either with Generic attributes or an Application Domain Extension (ADE).

Building Information Modelling (BIM) is a 3D modelling procedure that describes the geometric and semantic properties of a building and monitors its life-cycle. BIM complies with the IFC standard which is a standardized open data model developed by the international organization buildingSMART (Building SMART International, 2007). IFC supports Level of Development (LOD) 100 to 500. For the purposes of this paper, the IFC Buildings consist of the following elements (adapted from Building SMART International, 2007):

- WallStandardCase: the component representing the wall surface of a model, enclosing it vertically.

- Slab: the component representing the ceiling or the floor of the model, enclosing it horizontally.

- Space: a non-geometric representation of the free space of the building.

- Door \& Window: elements that close openings on a wall surface.

- $\quad$ Railing, Stair \& Stairflight: contain the elements required to form a stair.

- Furnishing element: contains the furniture of the building.

\subsection{Approaches to Data Integration \& Interoperability}

Current approaches to $\mathrm{BIM}^{1 / 3 \mathrm{D}}$ GIS data interoperability can be classified based on the conversion process (following ElMekawy, 2012) in relation to each method's approach to manipulate geometry and semantics. To this end, they can be identified as unidirectional, extensions to CityGML and using neutral formats. Each of the above three classes is briefly described below.

2.2.1 Unidirectional Integration Methods: With regard to examining the geometric differences between the two Standards, Nagel (2007) presents a unidirectional conversion algorithm for the automatic generation of valid CityGML LoD 1 models, by simplifying the geometry of the model and extruding the third dimension. Limitations include not examining higher LoDs and excluding semantics from the conversion. Ellul et al. (2015) investigate a unidirectional conversion from BIM to GIS elaborating further on the differences of the geometries between the two standards and especially on the issues that arise for the conversion of space geometries and boundaries. Furthermore, Boyes et al. (2017) examine the conversion of IFC geometries to $3 \mathrm{D}$ GIS via FME, summarizing the challenges that occur due to limited support for Constructive Solid Geometry (CSG) by FME Workbench, while the generated geometries from Trimble SketchUp are a workable alternative solution.

In terms of investigating both geometry and semantics between the two Standards, Isikdag and Zlatanova (2009) propose a unidirectional framework for generating CityGML models using BIM in two stages: semantic mapping and geometry simplification. Nagel, Stadler et al. (2009) propose reconstruction methods for $3 \mathrm{D}$ models by splitting the process in two stages: (i) conversion of the 3D model to CityGML based on specific spatio-semantic principles, (ii) CityGML to IFC. The results determine significant challenges in handling geometry between the two standards, since CityGML supports exclusively boundary-representation (b-rep), while IFC is more flexible. El-Mekawy (2012) analyzes the semantic mapping between IFC and CityGML and concludes that there are noticeable differences with the geometric and semantic structure of CityGML. Donkers et al. (2016) present an automatic process for converting an IFC model to a LoD 3 CityGML that is evolved in three stages: semantic filtering and mapping, geometric transformations and geometric and semantic refinements. The generated models contain only the semantic information that is relevant with the CityGML standard, thus the additional IFC semantic properties are discarded. The necessity of expanding the conversion not only to CityGML LoD 4 models but also to other city objects such as tunnels, bridges and roads is noted. Geiger (2015) demonstrates the importance of generalizing IFC models, aiming to reduce geometric and semantic redundancy and facilitate the extraction of a LoD 1-3 representation, while the need for LoD 4 models remains. Furthermore, questions arise with regard to the efficient mapping not only of geometry but also semantics. Zlatanova et al. (2013) state that one of the limitations of the conversion between IFC and CityGML is the missing semantics that are stored in enriched IFC models, which is one of the challenges this paper aims to address.

\footnotetext{
${ }^{1}$ In the context of this paper, BIM refers to semantically enriched $3 \mathrm{D}$ modelling and focuses on the semantic aspects in particular. Thus, issues relating to parametric modelling of geometry or life-cycle management are not considered.
} 
2.2.2 Integration via Extensions to CityGML: The integration between IFC and CityGML is also feasible by extending CityGML via an Application Domain Extension (ADE). Cheng et al. (2013) propose a framework that supports bidirectional translation between IFC and CityGML at different Levels of Detail by developing a CityGML ADE named Semantic City Model (SCM). De Laat and van Berlo (2011) develop the CityGML GeoBIM extension that allows the semantic enrichment of a CityGML model with IFC data and is implemented on BIM server. The limitations of those approaches involve the generation of huge CityGML files in order to process the IFC conversion as well as the excess use of more IFC Entities than those required in a CityGML model, hence complicating the management and discouraging the implementation of a 3D city model in different application fields.

2.2.3 Conversion to intermediate neutral formats: Another approach to $3 \mathrm{D}$ data integration is the implementation of a new model that serves the role of a neutral intermediate format aimed to assist on a specific purpose. Benner et al. (2005) presented a 3D building model for urban applications. The QUASY system presents many similarities with CityGML but it is more flexible than CityGML due to the application of Quvariants (Liu, 2017), since it is semantically enriched with extensions such as storeys, passages and opening objects. IndoorGML is another framework developed by the IndoorGML Standard Working Group in an OGC GML 3.2 application schema. It facilitates indoor navigation but can also provide valuable insight to the IFC and CityGML. More specifically, the capability of providing indoor data to the aforementioned standards can enhance its role in the integration between IFC and CityGML. El-Mekawy (2012) proposed another framework: Unified Building Model (UBM) which encapsulates both the geometry and semantic aspects of CityGML and IFC, thus facilitates a smoother communication of the two standards.

\subsection{Available data conversion tools}

In general, a semi-automatic process for converting BIM and GIS data is developed by implementing an Extract Transformation-Load process (described in Liu, 2017). The mapping process during ETL is characterized by its capability to manipulate each entity separately, offering a wide degree of freedom to the user and also facilitating a fully customized conversion between BIM and GIS (Liu, 2017). There are numerous conversion tools available that convert IFC to CityGML such as BIMserver, KIT IFCExplorer and Feature Manipulation Engine (FME) by Safe Software (Donkers et al, 2016). BIMserver and IFCExplorer are able to convert successfully the IFC Geometry but lack semantic mapping (Donkers et al., 2016). One tool that can address both geometry and semantic conversion tasks is Feature Manipulation Engine (FME) (Safe Software, 2017) which allows the geometries and semantics to be maintained, addressing the issue of lossy conversions especially in terms of semantic information that often arises in unidirectional methodological approaches (Zlatanova, 2013). Also, the capability to manipulate each entity separately during the conversion process results in tackling issues that automatic methods occasionally overlook (Donkers et al., 2016) and may affect negatively the generated model. However, it remains a challenge to convert IFC models to valid CityGML LoD 4 since barriers such as the geometric inaccuracy and semantic incoherence of boundary surfaces cause issues when validating the compliance of the generated models with the
CityGML format, leading to the requirement to improve the existing conversion workflows. Lastly, the bidirectional capabilities of the software render it a valuable tool for future investigation in order to fully understand the communication between the two standards.

\section{DATA \& SOFTWARE}

In order to more realistically emulate the input data quality that would be available in a real-world situation, the proposed conversion workflow is tested on real data. The architectural plans of School of Electrical Engineering in the campus of National Technical University of Athens are provided in four files of $2 \mathrm{D}$ CAD format due to the significant area of the modelled building ${ }^{2}$. The files include side views utilized to form the element views in Revit and plan views to create the model. The architectural plans of the second model located in the municipality of Chalandri are provided in 2D PDF format and represent the plan views of the building. Both models are generated from scratch in Autodesk Revit 2017 utilizing the 2D plans. The implemented software tools are: AutoCAD Civil 2017, AutoCAD Map 2017, Autodesk Revit 2017 and Trimble SketchUp 2017. For spatial processes QGIS 2.14 is applied and the conversion workflow is developed in FME. Model visualization is performed in FZK Viewer.

\section{METHODOLOGY}

Figure 1 shows an overview diagram of the data conversion workflow from IFC $2 \times 3$ to CityGML LoD 4 . In brief, firstly the model is designed utilizing BIM and then exported in IFC $2 \times 3$. Next, it is converted to CityGML LoD 4 inside FME and is enriched with additional semantic information.

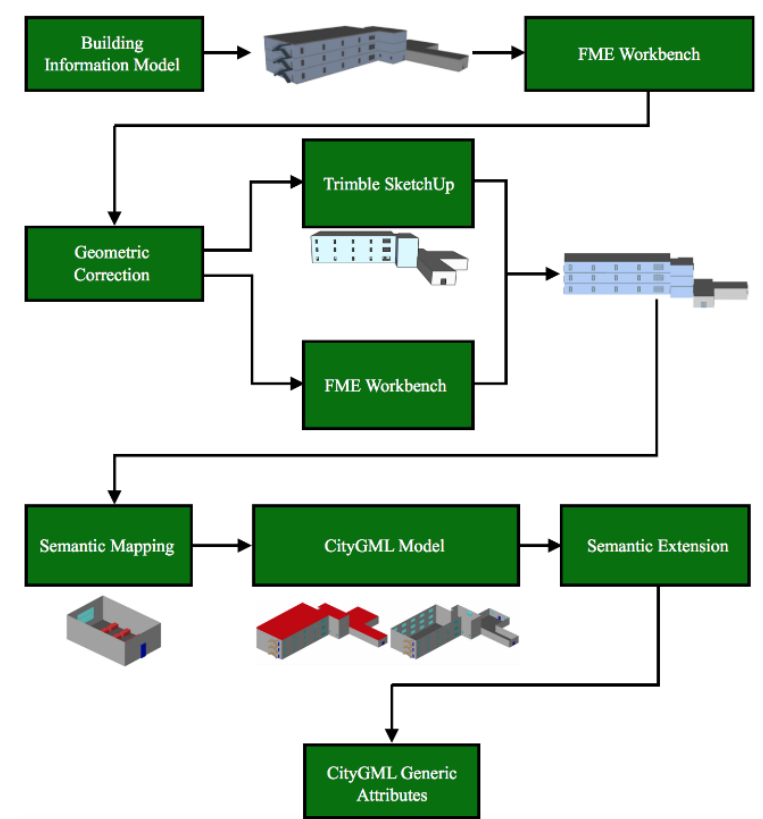

Figure 1: Workflow of the process

Sections 4.1 and 4.2 present the modelling and conversion phase respectively. The IFC models are generated from scratch following a standard BIM creation process.

\footnotetext{
${ }^{2}$ Note that while BIM may be created directly for new buildings, in the case of existing buildings CAD files offer an excellent source of based data for BIM creation and have been used here to demonstrate this process.
} 
To evaluate the advantages and limitations of the developed methodology, the IFC models are augmented with semantic information which is associated with geometric and nongeometric objects, such as spaces. This semantic enrichment intents to investigate how IFC handles this information and how this information can be transferred to CityGML.

\subsection{Modelling in BIM}

The BIM models are designed from scratch in Revit by making use of the geometric information provided by the architectural plans and then are enhanced with semantic detail to better represent the output of a real-world BIM. In order to geolocate the models, true orthophotos of the area are georeferenced in QGIS 2.14 and then imported in AutoCAD Map 2017 to digitize the boundaries of the model and link them with the Revit models in order to acquire their coordinates. The coordinate system of the model is the Greek Grid Reference System '87 (GGRS '87). Next, the element views are set to define the top and bottom constraints of the models' elements. When the boundary surfaces and interior of the building are modelled, openings and multiple components are installed. The installed elements (fig. 2) are different types of doors and windows depending on their usage, stairs connecting the floors of the building and classroom equipment such as desks and chairs in order not only to emulate a detailed building model, but also to enable an investigation of the conversion limitations and challenges that may or may not occur during the conversion of IFC to CityGML at LoD 4. Interior elements and more specifically building furniture are not included in the original architectural plans but in order to test the capabilities of the conversion workflow they are modelled as part of the BIM process utilizing Revit's component family.

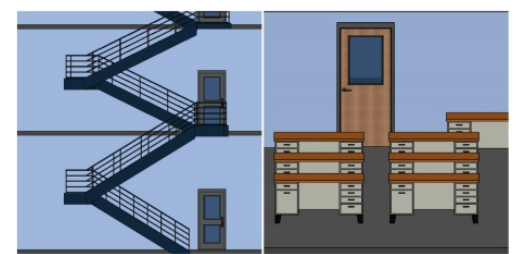

Figure 2: Model enrichment with components in BIM

The models are semantically enriched with information that is stored either as properties of surfaces, or properties of objects without geometry, such as spaces, in order to test the outputs in different conditions. Boundary surfaces such as walls, roof and floor are assigned the material which they are made of. Openings, such as windows and doors include information with regard to the main and secondary construction material and their reflectivity. The second model, is additionally enriched with legal information. Autodesk Revit provides the capability of setting specific areas as rooms. Within this context, four rooms are created and the semantic enrichment takes place by creating corresponding schedules. Inside those schedules, cadastral information is assigned and the following items are generated: "Cadastral Code Number", "Ownership Properties", "Land Use" and "Type of Weight". The aforementioned properties are assigned on a semantically enclosed space, enabling the examination of preserving semantic information that is located in non-geometric elements. The BIM models are exported to IFC $2 \times 3$ format.

\subsection{Geometric correction}

Geometric correction is performed in two phases since FME's current transformers are not able to generate models that comply geometrically with the CityGML LoD 4 specifications. Specifically, issues arise regarding the conversion of LoD 4 models with complexity in their boundary surfaces, such as "niches" in the walls. Therefore, IFC WallStandardCases and IFC Slabs are converted in Trimble SketchUp and the rest of the IFC entities are converted in FME Workbench.

4.2.1 Converting Wall \& Slab Data in Trimble SketchUp: Firstly, IFC WallStandardCase and the IFC Slab are extracted via a separate workflow and written in skp format. The workflow initially extracts the "Body" geometry of the IFC entities and then coerces the solids to composites surfaces. Afterwards, the overlapping geometries inside the model are detected and removed and a unique ID is assigned to the remaining elements. The model is inserted in Trimble SketchUp and the correction of the geometries is performed by utilizing specific tools such as extruding objects and grouping components. Firstly, objects such as walls, are represented as multisurfaces and not solid geometries which facilitates the geometric correction by implementing the "erase" tool in order to discard the redundant geometries. Furthermore, previous research (Dimopoulou et al., 2014; Floros et al., 2015) demonstrates that Trimble SketchUp is able to generate CityGML models via an FME Workbench. The extraction of the geometries in Trimble SketchUp is able to overcome and solve issues such as unintentional model errors or disjointed surfaces (Donkers et al., 2016) in order to ensure geometrically concrete surfaces and objects. This process aims to fix the geometric accuracy of the wall surfaces and the slabs in LoD 4 models since the roof or the ground surface should be further decomposed in ceiling and floor surfaces respectively. In order to solve it, the roof surface is "moved inside" and the attached wall surface is extruded up to the level of the roof surface. The model is inserted back in FME Workbench as a skp file to proceed with the conversion to CityGML.

4.2.2 Processing in FME Workbench: The geometric correction of the rest of the IFC Elements is summarized as follows: firstly, each object is decomposed in its elemental parts and the geometry with the trait "Body" is extracted. Then, the geometry is converted to a composite surface, pending semantic mapping with the CityGML Entities.

\subsection{Semantic Mapping}

The mapping between IFC and CityGML entities follows the principles highlighted by Laat and v. Berlo (2011), while the considered IFC Entities are presented in fig. 3. Additionally, during the semantic mapping between the two standards it is taken into consideration that an IFC entity may contain other IFC entities.

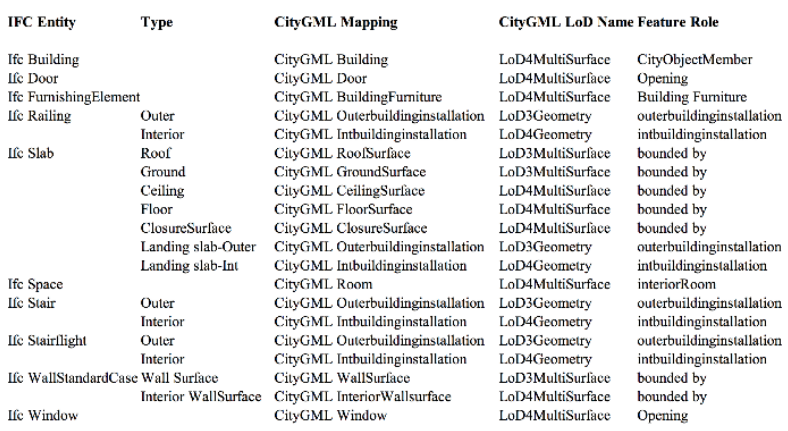

Figure 3: Semantic mapping from IFC to CityGML LoD 4 
IFC Buildings are semantically similar to CityGML Buildings, which are formed by boundary surfaces, such as walls, ceilings and floors. IFC Space is used to model the interior free space in a Building and corresponds to the Room in CityGML. The Rooms in CityGML are compiled of CeilingSurfaces, InteriorWallSurfaces, FloorSurfaces and Closuresurfaces. The CityGML Rooms are linked to the CityGML Buildings using appropriate identifiers and a parent-child relationship.

Next, the extracted IFC WallStandardCase and IFC Slab which are created and geolocated in Trimble SketchUp, are semantically linked (via a unique ID that is conserved through both processes) back to the CityGML Building.

The semantic mapping of the interior surfaces is achieved by joining every surface (CeilingSurface, InteriorWallSurface, FloorSurface and ClosureSurface), with the appropriate Room. A noticeable semantic difference at this stage, is that the "IsExternal" semantic property of IFCWallStandardCases is not always accurate (Donkers et al., 2016). This is depicted in figure 4, where a part of the wall is exterior, while the rest of it is part of the interior building. The proposed methodology considers such issues and addresses them by mapping the objects to the appropriate CityGML surfaces.

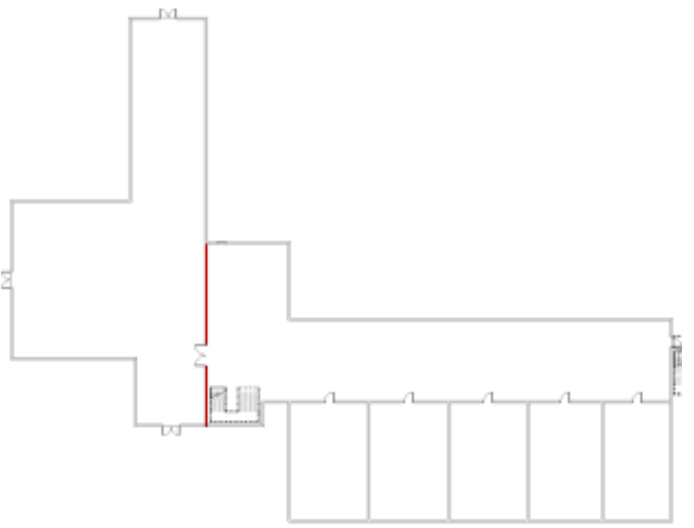

Figure 4: Highlighted (red) part of the wall is considered as exterior wall

In CityGML, if the surface of the wall contains openings, then this relationship must also be depicted semantically (Open Geospatial Consortium, 2012, p. 12). Thus, a common attribute is created for both the surface and the opening and then, those two features are merged to be semantically linked. Additionally, since the generated CityGML model is LoD 4, the openings must also be semantically connected with the interior surfaces in order to enable a connection of adjacent rooms, by performing the same linking process.

Regarding the semantic mapping of IFC Furnishing Elements, a connection between the furniture and the room they belong to must be established. This is feasible by creating attributes that link each element with the relevant Room. Lastly, the IFC Stair Flight, Railing and Stair need to be semantically mapped as outer and interior building installations. With regard to the outerBuildingInstallation, the attribute gml_parent_id matches the gml id of the Building. In CityGML, an interiorinstallation can belong to either the whole building or to a specific room (Open Geospatial Consortium, 2012, p. 76). For the purposes of this paper, the interior building installations have parentchild relationship with the CityGML Building (parent).

\subsection{Semantic enrichment of the models}

The semantic extension of the CityGML model is feasible by modifying the CityGML writers inside the FME Workbench. More specifically, each extended CityGML feature type must implement the relevant IFC attribute alongside the data type of the information (i.e. integer, Boolean, string). This approach is used to enhance the first CityGML model with information about surface material, and the second with legal information as described in section 4.1. For the latter, the use of IFC Space and CityGML Room is mandatory.

\section{RESULTS}

\subsection{Visualization of Conversion Results}

The generated models support multi-LOD modelling as structured by CityGML, thus enabling the modelling and visualization of an object in different LoDs, while maintaining its attributes and characteristics. Additionally, the co-existence of multiple LoDs in the same CityGML dataset is supported (Open Geospatial Consortium, p. 11, 2012). For instance, while the WallSurface could be generated as a LoD 4 Multisurface geometry, it is generated as a LoD 3 Multisurface geometry. Fig. 5 and 6 illustrate the generated CityGML models in LoD 3 and 4.

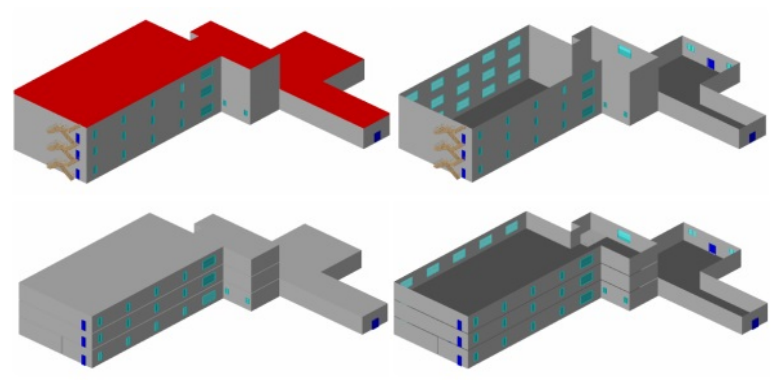

Figure 5: CityGML LoD 3(Top) \& CityGML LoD 4 (Bottom) models of Building 1

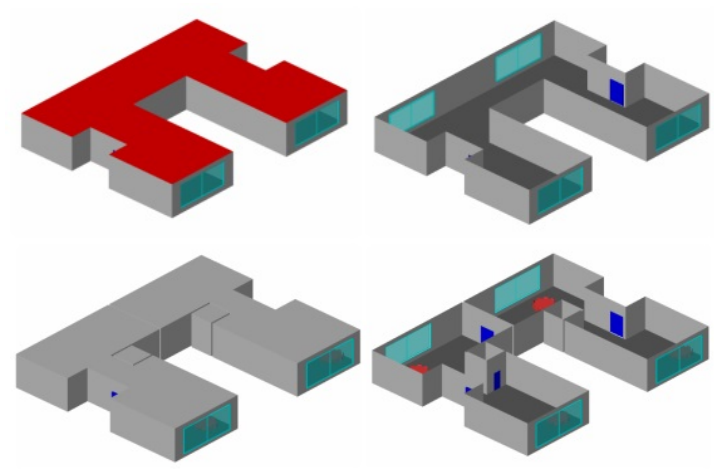

Figure 6: CityGML LoD 3(Top) \& CityGML LoD 4 (Bottom) models of Building 2

The geometric validation of the models is performed in the environment of FME Workbench, by filtering the generated models based on the following criteria: duplicated geometries, non-planar surfaces and overlapping geometries.

The semantic coherence of the models is examined via FZK Viewer (fig. 7). The interior space of the buildings is mapped to form the CityGML Room feature, which is formed by the boundaries of CityGML InteriorWallSurfaces, CeilingSurfaces and FloorSurfaces. Additionally, the 
openings (doors \& windows) are semantically connected to the relevant interior wall surfaces they belong to. Lastly, elements that are mapped as CityGML BuildingFurniture are placed inside the relevant Room.
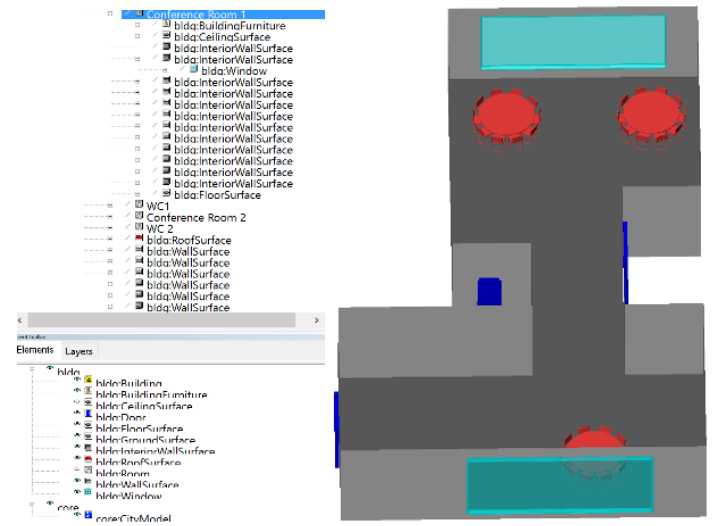

Figure 7: Semantic examination of the CityGML LoD 4 model in FZK Viewer

\subsection{Semantic extension with Generic Attributes}

The surface material is an important attribute in a building. However, IFC is designed for AEC purposes and does not support texture visualization (De Laat and v. Berlo, 2011). Within Autodesk Revit, the wall surfaces are assigned the "brick" material, which is successfully visualized, but during the export to IFC format the texture of the surfaces is lost. However, as discussed in Section 4.4 it is possible to maintain this type of information as semantic information (fig. 8).

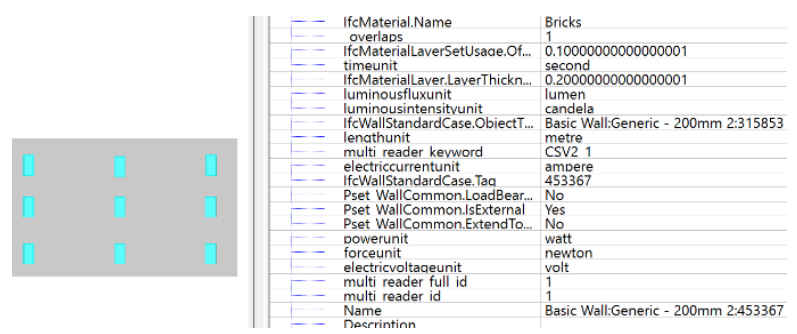

Figure 8: IFC material of walls transferred to CityGML LoD 4 model

Figure 9 illustrates the extension of a CityGML model with semantic information that is assigned to non-geometric objects in IFC and is related to the legal aspect of a building such as its cadastral number, use, restriction (type of weight) and ownership status. CityGML supports this extension by providing the attributes citygml class, usage and function. The citygml_class uses the value "Commercial", following the standard, which mandates that the use of Generic attributes should be implemented where there are no relevant extensions of the CityGML that can fill that purpose (Open Geospatial Consortium, 2012, pp.146-148). The Generic Attributes retain the naming and values created during BIM process.

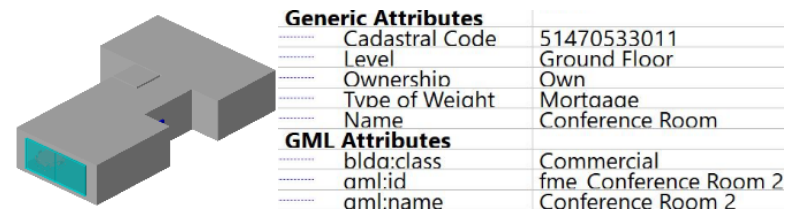

Figure 9: Legal properties transferred from IFC to CityGML LoD 4 model

\section{DISCUSSION}

The developed methodology can generate valid CityGML LoD 4 models by implementing ETL process and more specifically a data conversion workflow in FME Workbench. Furthermore, it utilizes the multi-LOD concept of CityGML and represents the features in the correspondent LoD of CityGML Standard. This could prove particularly useful during the management of the model, since an extraction of certain elements based on the LoD within a spatial database is feasible. Additionally, the modelling process in BIM highlights the capabilities of adding multiple information to different elements regardless of whether they contain geometry or not, and then migrating the information to CityGML LoD 4 without loss, which according to multiple sources (Zlatanova, 2013; De Laat and v. Berlo, 2011) constitutes a great challenge for efficient interoperability between the two standards. Moreover, the different software environments during the conversion process can benefit the generated CityGML model by fixing errors that affect the representation and implementation of the generated model such as a door that misses multiple surfaces due to the fact that the IFC properties and elements are either damaged or missing elements in the first place. Another example is the fact that IFC solids can overlap which can be a common mistake since in BIM all components are placed based on the set elevation views and an unintentional error is possible. Similarly, semantic information can be inspected and modified to fix unintentional errors that may occur during the modelling process, since they can be stored in a separate file. Lastly, the proposed methodology is able to convert models in lower LoDs, reducing significantly the complexity in the conversion workflow and be able to contribute to application fields that require 3D models in lower LoDs.

Regarding disadvantages, in complex buildings that need to be converted in CityGML LoD 4 models, the overall conversion process can be time-consuming. The separation and manipulation of the entities in order to generate a LoD 4 model is more challenging compared to a LoD 3 model since the interior of a building encloses more geometric and semantic information than the exterior. Moreover, the familiarity of a user with FME Workbench or Trimble SketchUp should not be implied and thus it may delay significantly the conversion process. Also, in terms of the conversion process to a CityGML LoD 4 model, there is the limitation of manipulating adjacent surfaces between building parts, since in IFC the walls are solid geometries and this conversion cannot be overcome by the proposed methodology. While LoD 4 conversion is possible, manual intervention is still required.

\subsection{IFC to CityGML LoD 4}

In order to convert an IFC model to CityGML LoD 4 there are a number of interoperability issues that need to be considered. Failure to take these into account could result in 'holes' in the CityGML model due to unconverted information. Examples include:

- Is a slab a floor, ceiling or both? The horizontal surfaces that forms the floor and the ceiling of each storey in CityGML, contains different attributes in BIM. More specifically, the solid geometry that is designed as a roof object, is solely a roof of the model. In contrast, in CityGML the lower part is assigned a CeilingSurface feature while the higher part is assigned a RoofSurface or a FloorSurface. These semantic differences are examined 
at the stage of semantic filtering and mapping, since a roof surface that is mapped as floor will contain semantic information that corresponds to the primary feature.

- Is a slab part of the stairs or a separate floor entity? A complex of stairs might be categorized in multiple IFC entities not only semantically, but also geometrically. For example, some surfaces are labelled as slabs, while in BIM they are labelled as landing surfaces, which are parts of the stairs.

- Do the boundaries of a slab intersect with the interior wall? In LoD 4 the geometry must comply with the CityGML standard and the slabs should be modelled based on the exterior of the wall boundaries. In case the IFC model is structured otherwise, the conversion workflow is able to handle those changes and produce geometrically valid CityGML models.

In all cases, providing clear guidance to the BIM modeler e.g. 'a partial landing where the stairs turn should be classified as a stair component, but if the landing is at the level of a main floor of the building it a floor slab should be used' will help to remove these ambiguities.

\subsection{Automation of the workflow}

The proposed conversion workflow can be utilized to convert different IFC models since it includes the required CityGML feature types and properties, but the process is not straightforward and must be adapted to each new situation. The results can be examined based on the targeted LoD:

For LoD3, the conversion is relatively straight-forward. If the model contains additional IFC entities, the workflow can be modified appropriately to deal with, for example, a specific mapping between an IFC object and a corresponding CityGML object, with the overall steps regarding the geometry adjustment and semantic mapping remaining unchanged. As the LoD 3 conversion discards the interior of the model, the workflow does not require significant modification, however the geometric correction in Trimble SketchUp still needs to be implemented for the exterior surfaces to properly tackle the limitation of converting geometries in FME Workbench.

In an LoD 4 conversion, however, the process is more complicated since the CityGML Rooms are defined by ceilings, floors and interior walls. In BIM models, a room is specified by tagging a specific area that is enclosed by interior walls. The floor and ceiling of the building is usually represented as a single surface; thus, the complexity of the conversion workflow increases since the intersection of the interior wall surface with the ceiling and the floor needs to be located and fixed inside Trimble SketchUp. The conversion from IFC to CityGML LoD 4 also requires the implementation of an external software environment -Trimble SketchUp- in order to be successful. The reason is that Trimble SketchUp supports B-Rep geometry which is also supported by CityGML and can be manipulated inside FME Workbench. On the other hand, existing FME transformers struggle handling IFC's solid geometries. With regard to the field of semantic mapping, there is no requirement for substantial changes. As long as the IFC entities are properly distinguished they are automatically matched with the corresponding CityGML entities. Lastly, regardless the LoD of the model, a manual georeference in a GIS is required.

\subsection{Applying the Results in a Real-World Context}

The results demonstrate that BIM could become a valuable source of both semantic and geometric information in order to enrich a CityGML model in terms of the Building class. While BIM models are currently not optimally designed to store legal information, the above research has demonstrated that it is possible to extend the model to include information related Cadastral and Land Administration systems. Additionally, the same workflow can be adjusted to fit the special requirements and limitations of application fields that serve different purposes - i.e. the preservation of semantic information in non-geometric objects can be applied in fields such as environmental protection, sustainability and energy monitoring. Within this context, the application field that the model is generated for should be considered as part of the process of identifying best practice guidance for BIM modelers.

\section{CONCLUSIONS \& FUTURE RESEARCH}

The work presented demonstrates the potential of using BIM models as a data source for CityGML 3D City Models, and hence as a more detailed underpinning framework to utilize technologies such as Smart Cities and Internet of Things. It is not an automatic process, and when compared to other methodologies that generate LoD 3 and lower CityGML models, it is less time efficient. However, the different phases of the conversion provide opportunities for the user to inspect the converted model in order to locate and fix issues that cannot be tackled by automatic conversion methodologies. Furthermore, it extends successfully the generation of CityGML models to the higher LoD, allowing the introduction of more semantic information at source - i.e. within the BIM. The process then successfully preserves and transfers semantics to CityGML models enabling use in specific application fields.

The approach described in this paper will also maximise the financial benefit gained from costly BIM creation by reusing the expensive BIM data beyond the construction phase of a project. This feeds into the wider agenda for BIM to be used over an entire building life cycle.

\subsection{Future Work}

Future research should investigation of different types of semantic information that can be implemented within a BIM model for different application purposes, in particular with consideration as to when it is most appropriate to enrich a model - e.g. what information is available and relevant when designing the BIM and would naturally form part of a BIM creation workflow, versus what information should be added at a later stage, following on from conversion.

Technically, the main challenge to be addressed is to reduce the amount of manual intervention. Currently issues in terms of handling the geometry are tackled by manipulating it separately in Trimble SketchUp, due to certain limitations of the FME Workbench. However, it is anticipated that those limitations can be tackled by utilizing the API of the software or by implementing scripts.

More importantly, to facilitate further automation it is fundamental that the BIM is created to a set of guidelines that not only match the requirements of the construction process but also allow the IFC/CityGML interoperability issues to be 
tackled by standard conversions. The work described in this paper provides a useful input into this, highlighting the geometric and modelling differences between wall and slab surfaces, as well as the semantic issues that arise when converting multiple IFC elements to a single CityGML class. In the future, a full list should be developed in collaboration with all stakeholders - building designers, BIM model creators, and users of the resulting CityGML data, in order to identify a solution that best fits existing requirements and workflows.

\section{REFERENCES}

Batty, M., Axhausen, W., K., Giannotti, F., Pozdnoukhov, A., Bazzani, A., Wachowicz, M., Ouzounis, G. \& Portugali, Y., 2012. Smart cities of the future. The European Physical Journal Special Topics, pp. 481-518.

Benner, J., Geiger, A. and Leinemann, K., 2005. Flexible Generation of Semantic 3D Building Models. In: Proceedings of the 1st international workshop on next generation $3 D$ city models, pp. 17-22, Bonn, Germany.

Boyes, G., Ellul, C. and Irwin, D., 2017. Exploring BIM For Operational Integrated Asset Management- A Preliminary Study Utilising Real-World Infrastructure Data. In: ISPRS Annals of the Photogrammetry, Remote Sensing and Spatial Information Sciences, Volume IV-4/W5. 12th 3D Geoinfo Conference 2017, Melbourne, Australia.

Boyes, G., Thomson, C. and Ellul, C., 2015. Integrating BIM and GIS: Exploring the use of IFC space objects and boundaries. In: Proceedings of the GISRUK 2015, Leeds, UK, 15-17 April 2015; University of Leeds: Leeds, UK.

Building SMART International, 2007. Industry Foundation Classes (IFC), IFC2x3. Available from: http://www.buildingsmart- tech.org/specifications/IFCreleases/summary.

Cheng, J. C. P., Deng, Y. and Du, Q., 2013. Mapping between BIM Models and 3D GIS City Models of Different Levels of Detail. In: Proceedings of the 13th International Conference on Construction Applications of Virtual Reality (CONVR 2013), London, UK, 30-31 October 2013.

De Laat, R. and van Berlo, L., 2011. Integration of BIM and GIS: The development of the citygml GeoBIM extension. In: Advances in 3D Geo-Information Sciences. Springer: New York, NY, USA, 2011; pp. 211-225.

Dimopoulou, E., Tsiliakou, E., Kosti, V., Floros, G. and Labropoulos, T., 2014. Investigating Integration Possibilities between 3D Modeling Techniques. In: Proceedings of 9th International 3D GeoInfo Conference, Nov. 2014, Dubai, United Arab Emirates.

Donkers, S., Ledoux, H., Zhao, J., \& Stoter, J., 2016. Automatic conversion of IFC datasets to geometrically and semantically correct CityGML LOD3 buildings. Transactions in GIS, 20(4), 547-569. http://doi.org/10.1111/tgis.12162

El-Mekawy, M., Östman, A. and Hijazi, I., 2012. An Evaluation of IFC-CityGML Unidirectional Conversion. In: International Joural of Advanced Computer Science and Application.
Floros, G., Tsiliakou, E., Kitsakis, D., Pispidikis, I. and Dimopoulou, E., 2015. Investigating Semantic Functionality of 3D Geometry for Land Administration. In: Advances in 3D Geoinformation, Lecture Notes in Geoinformation and Cartography. Springer: Kuala Lumpur, Malaysia, 2015, pp. 247-274. doi: 10.1007/978-3-319-25691-7_14

Geiger, A., Benner, J. and Häfele, K. H., 2015. Generalization of 3D IFC building models. In: 3D Geoinformation Science. Springer: NY, USA, pp. 19-35.

Jusuf, K., S., Mousseau, B., Godfroid, G. and Vincent Soh, H., J., 2017. Path to an Integrated Modelling between IFC and CityGML for Neighborhood Scale Modelling. In: Urban Science, Vol. $\quad 1, \quad$ Issue 3. https://doi.org/10.3390/urbansci1030025.

Isikdag, U. and Zlatanova, S., 2009. Towards Defining a Framework for Automatic Generation of Buildings in CityGML using Building Information Models. In: Lee J. and Zlatanova, S. (Eds.) 3D Geoinformation Sciences, pp.79-96, Springer Berlin Heidelberg.

Liu, X., Wang, X., Wright, G., Cheng, J., Li, X. and Liu, R., 2017. A State-of-the-Art Review on the Integration of Building Information Modeling (BIM) and Geographic Information System (GIS). In: ISPRS International Journal of Geo-Information 6(2), pp. 53.

Nagel, C., 2007. Conversion of IFC to CityGML. Meeting of the OGC 3DIM Working Group at OGC TC/PC Meeting, Paris (Frankreich), July 2007.

Nagel, C., Stadler, A. and Kolbe, T. H., 2009. Conceptual requirements for the automatic reconstruction of building information models from uninterpreted 3D models. In: International Archives of Photogrammetry, Remote Sensing and Spatial Information Sciences, 38.

Open Geospatial Consortium, 2012. OGC City Geography Markup Language (CityGML) Encoding Standard 2.0.0. Technical report.

Arroyo Ohori, K., Biljecki, F., Diakite, A., Krijnen, T., Ledoux, H. and Stoter, J., 2017. Towards An Integration Of GIS And BIM Data: What Are The Geometric And Topological Issues? In: ISPRS Annals of the Photogrammetry, Remote Sensing and Spatial Information Sciences, Volume IV-4/W5. 12th 3D Geoinfo Conference 2017, Melbourne, Australia.

Safe Software. IFC LOD 300 to LOD 4 CityGML. URL: https://knowledge.safe.com/articles/1025/bim-to-gisintermediate-ifc-lod-300-to-lod-4-cityg.html , Accessed on 03/09/2017.

Zlatanova, S., Beetz, J., Boersma, A., Mulder, A. and Goos, J., 2013. 3D spatial information infrastructure for the port of rotterdam. In: Proceedings of the International Workshop on "Global Geospatial Information", Novosibirsk, Russia, 25 April 2013. 